2019-04-16

\title{
Generalisation of Anthropomorphic
}

\section{Stereotype}

\section{Zanatto, Debora}

http://hdl.handle.net/10026.1/13918

10.1007/s12369-019-00549-4

International Journal of Social Robotics

Springer Verlag

All content in PEARL is protected by copyright law. Author manuscripts are made available in accordance with publisher policies. Please cite only the published version using the details provided on the item record or document. In the absence of an open licence (e.g. Creative Commons), permissions for further reuse of content should be sought from the publisher or author. 


\title{
Generalisation of Anthropomorphic Stereotype
}

\author{
Debora Zanatto - Massimiliano Patacchiola · Angelo Cangelosi · Jeremy \\ Goslin
}

the date of receipt and acceptance should be inserted later

\begin{abstract}
Anthropomorphic projection can bring familiarity, confidence and simplicity to our interactions with unknown agents showing a human-like resemblance or behaviour. This study examined whether this projection is generalised beyond the individual agent to encompass others of similar type, even if they might be lacking the requisite human-like features. In an experiment participants had to accept or reject recommendations from two robots that had more or less humanlike forms and behaviours. It was found that participants were more likely to trust the judgements of a less-human like robot if they had previously interacted with an anthropomorphic variant. Importantly, this effect was found to be symmetric, with trust in anthropomorphic robots reduced if participants had previously interacted with a less human-like variant. These results showed that we generalise our initial attributions across agent categories, a finding with potential application in helping trust and acceptance of complex technological agents.
\end{abstract}

Keywords Stereotype Activation - Anthropomorphism · Trust $\cdot$ Human-Robot Interaction

This material is based upon work supported by the Air Force Office of Scientific Research, Air Force Materiel Command, USAF under Award No, FA9550-15-1-0025.

Debora Zanatto · Massimiliano Patacchiola · Angelo Cangelosi, School of Computing, Electronics, and Mathematics

Portland Square, Plymouth University, Drake Circus, PL4 8AA, Plymouth, UK.

E-mail: debora.zanatto@plymouth.ac.uk, massimiliano.patacchiola@plymouth.ac.uk, a.cangelosi@plymouth.ac.uk

Jeremy Goslin

School of Psychology

Portland Square, Plymouth University, Drake Circus, PL4 8AA, Plymouth, UK.

E-mail: jeremy.goslin@plymouth.ac.uk

\section{Introduction}

The tendency to anthropomorphise is fundamental to our psychology, a cross-cultural phenomenon with a history established in pre-historic art [17] and coined in ancient Greek philosophy (Xenophanes, as cited by Lescer [44]). It has been proposed [23] that a process of induction lies at the core of this highly prevalent phenomenon, one in which we use highly accessible knowledge on the self or human society as a base upon which we can explain or predict the unknown. Ascribing human intentions to unintentional agents, described as the intentional stance by Dennett [19], eases our uncertainty on how we can reason about or describe these agents. This stance would also make it easier to form social connections with non-human agents, extending our fundamental desire for social relationships [9] outside of humankind. Thus, anthropomorphism would appear to offer us a simpler, more familiar world that is richer in social interaction, giving us greater confidence in our interactions with it.

Historically, research in this area has been dominated by studies on the accuracy and functionality of our anthropomorphic beliefs $[15,33]$, as well as their potential benefits $[38,52,62]$. Another major area of research has been the examination of the factors that influence the extent to which people anthropomorphise non-human agents. Guthrie [31] noted the attribution of intentions and human-like mental states to literary characters were related to their morphological similarity to ourselves. This influence of morphology seems relatively straightforward; objects featuring human features $[6,14]$ or shape [3] are seen as more human-like than those that do not. Shape in particular, is used as one of the earliest cues to object animacy [30] in infancy. Additional features such imitation, communic- 
ation, or indicators of intentionality, can also influence the extent to which we anthropomorphise an agent [18]. However, similarity to human motion is probably the next most important cue after that of morphology [48]. Movement can give the impression of life [64], and even simple geometric figures can be anthropomorphised as long as the temporal relationship of their movements conforms to our own [34]. Previously mindless agents whose movements are slowed down or speeded up [49] to approximate human motion can gain mental states, while biologically meaningful motion can also lend intention to non-human agents [22].

Nevertheless, providing an anthropomorphic form to a robot might not be sufficient to facilitate people's interaction with it. Anthropomorphism in fact is not only limited to the appearance of a robot, and the robot social interaction with humans is also considered an important factor [37]. Investigations on non-verbal communication [12] for example, have focused mainly on gestures and gaze, reporting that robots capable of gaze shifting during social interactions are perceived as more enjoyable [1], and robots performing joint attention are rated as more competent [36].

The social consequences of anthropomorphic impressions have been widely studied in marketing by designing human-like features into a product or linguistic inference. For example, studies have similarly found that as a robot appears more human-like, people are more likely to appreciate them and work with them [28], follow their instructions, [40] and even empathise with them [56]. Moreover, it has been found that cars are evaluated more highly if they have human facial similarities [2], and people gambled more when slot machines were described in anthropomorphic terms [41,57].

These studies provide valuable insight into the range of factors may lead us to anthropomorphise a particular nonhuman agent. In this study we wanted to examine the specificity of anthropomorphic assignment. If we apply anthropomorphic status to a particular agent, do we also extend this human-like status to other exemplars of the same category of agent? Moreover, if there are variations in the distribution of anthropomorphic features across the category of agent, might this effect also lead us to extend human-like status to variants that may lack those features? As an example, the moon has visual features that approximate a human face which might lead us to anthropomorphise this particular astronomical body. Given that the moon is likely to be the first body of this type we would encounter, we may also generalise the anthropomorphic trait across hereto unseen astronomical bodies? If so, would this initial encounter increase the probability that we would also assign human-like status to encounters with other exem- plars of astronomical body even if, like Mars, they are lacking the requisite anthropomorphic features? Conversely, if the first astronomic body we were to view was Mars, would we then be less likely to anthropomorphise the moon, as we had already categorised astronomical bodies as being non-humanlike?

These potential effects would appear to be related to the concept of stereotype activation. Stereotypes allow to categorise and simplify the environment [45] and consequently, to associate traits and behaviours to individuals belonging to a social category [11]. Stereotype activation is a useful scaffold when the amount of information is not adequate to form an impression [67]. In this case, people drawn from their prior knowledge of a category to make decisions and generalise and extend that stereotype to similar exemplars. This stereotypical generalisation, like the tendency to anthropomorphise non-human agents, derives from the innate need of filling the gap between the lack of informations about the agent and the need to portray it and socially define it.

If anthropomorphic status is extended across members of an agent category then these studies suggest that the status assigned to the first exemplar of the category should increase the probability that the same status is assigned to subsequently encountered exemplars. Therefore, if the first exemplar of a new type of agent is assigned human-like status then this should transfer anthropomorphism in a future exemplar of similar type, and vice versa. In this study we tested this hypothesis by measuring the behavioural effects of anthropomorphism during interactions with an agent following the interaction with a less, or more, anthropomorphic initial exemplar. Non-human agents in our study were instantiated by two types of robot, each conforming to the same broad category of agent, but with exemplars provoking different degrees of anthropomorphism based upon their form and behaviour.

It could be considered that a human-like robot is the epitome of anthropomorphism, an explicitly constructed instantiation of anthropomorphic form, behaviour and function designed to enhance our interaction with and understanding of a highly obtuse technological agent. Studies have shown that robots can be highly effective in solicitors of anthropomorphic projection (for a review see [70]). This leads us to project our own social schemas onto them [26], especially when they have human faces or bodies [21], or are engaged in social roles [24]. Thus, if a robot has humanlike features it is perceived as anthropomorphic, and is expected to behave appropriately [58] and follow our social norms [61]. This social connection with anthropomorphic robots means that they are perceived as being 
more intelligent $[8,42]$, trustworthy $[32,66]$ and likeable [52], as well as attracting more visual attention [4] and empathy [56] than their less human-like variants. We even assign personality traits to the robot based upon idiosyncratic features of their height [65], gender features [53], face [68] or voice [51].

In a first attempt to study stereotype generalisation in HRI [69], we investigated whether prior experience with a human-like robot would lead people to increase the credibility of statements made by a lessanthropomorphic robot. Specifically, participants negotiated decisions on objects prices with either a more (iCub) or less- anthropomorphic robot (Scitos G5). While half of the participants only interacted with Scitos G5, the remaining half interacted with Scitos G5 only after having first interacted iCub. Results showed that credibility of the Scitos G5 was higher after participants were previously exposed to the iCub.

In this study we contrasted interactions with the NAO robot, a humanoid robot widely used in social robotics [43], with a Baxter robot, an industrial development robot [25]. Participants socially engaged with these two robots using a price judgement task developed by $\mathrm{Rau}, \mathrm{Li}$ and $\mathrm{Li}$ [54]. In this task participants were asked to make a monetary valuation on a common household object. This would be followed by a different valuation provided by the robot, after which the participants would be asked if they wanted to change their own valuation to agree with that of the robot. The primary measure of the robot perceived credibility was provided by frequency of the participant's willingness to change their valuation to match that of the robot. Based upon previous studies $[21,32,66,69]$ we would expect that the anthropomorphic form of the NAO robot would lend it greater social credibility than the Baxter. In addition to the manipulation of the physical form of the robots we also examined how the behaviour of the robots, specifically the use of social gaze, would affect their credibility. In human psychology gaze and joint attention provide cues to social trust $[10,46]$, effects that have also been established in Human-Robot Interactions (HRI) $[27,50,60]$.

Reiterating our original aims, we wished to examine if attributions of anthropomorphism are specific to an encountered non-human agent, or whether they were also generalised across other members of that agent type. To test our hypothesis we looked for evidence of the generalisation of anthropomorphic traits affecting the credibility of more and less human-like robots. While half of our participants played the price judgement game with the NAO robot first and the Baxter robot second, this order was reversed for the remaining participants. According to our hypothesis, the credib-

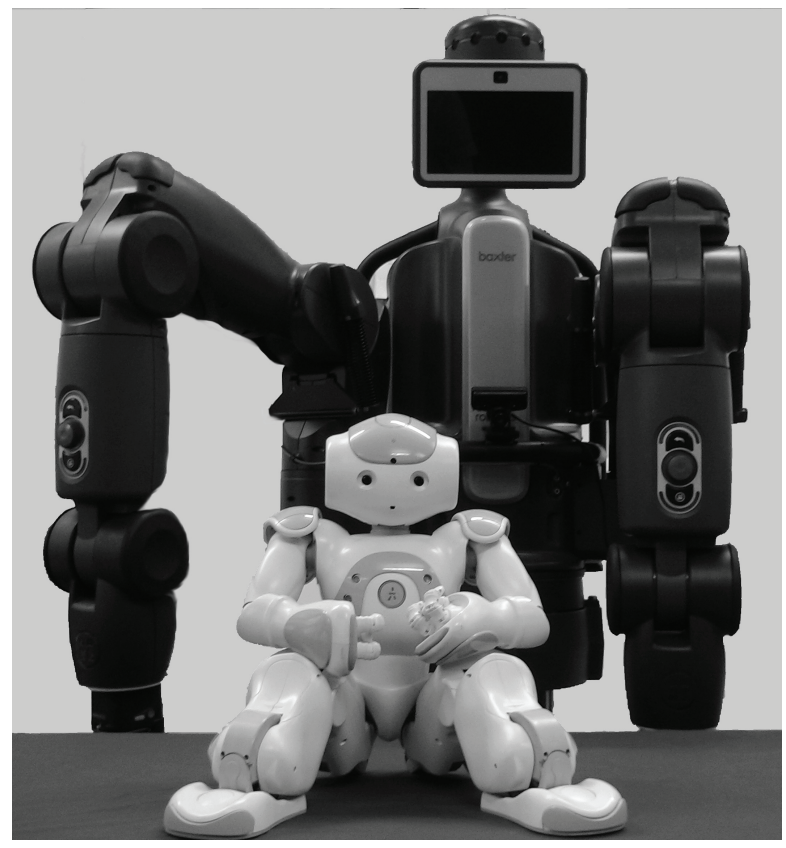

Figure 1 NAO robot (foreground) and Baxter robot (background)

ility of the Baxter robot should be higher when it is presented second, after participants have played with the NAO robot, than when it is presented first. Conversely, the credibility of the NAO robot should be lower when it is presented second, following interactions with the Baxter, than when it is presented first. Similar comparisons were also made for the gaze behaviour of the robots, comparing the use of a more anthropomorphic joint attention in the game to a condition where they simply stared fixedly ahead, expecting an increase of credibility in the first condition.

\section{Method}

During a price judgement task participants were asked to select from one of two valuations placed on common objects presented by a robotic compatriot. After a selection was made, the robot would voice its agreement or disagreement with the selected price and, if the robot did not agree with it, give them the chance to change their decision so that it would conform to its own. The willingness of participants to change their judgement was taken as a measure of the robot credibility. We used this task to examine how the form and behaviour of the robot would affect their perceived credibility, and whether their initial experiences with a particular robot would affect their future interactions with a different type of robot. Each participant made a separate series of price judgement interactions with two different types of robot, the human-like NAO and non- 
anthropomorphic Baxter robot. These robots could also be programmed to behave with either a social gaze or nonsocial gaze during their interactions. In the social gaze condition the robot was looking at the participants while making their selection, as well as when they had the chance to change decision. In the nonsocial gaze condition instead, the robot was always looking at the object on the table.

\subsection{Participants}

Thirty participants ( 5 males, 25 females) between 18 and 30 years (mean age $=23.20$ years, $\mathrm{SD}=2.10$ years) participated at the study. The sample size was based on Rau et al. [54] from which the task has been selected. Moreover, the sample size has been selected after the previous experimental results [69]. Participants were naïve as to the purpose of the investigation, with no previous experience with robot and gave informed written consent to participate in the study.

\subsection{Apparatus}

The NAO (Figure 1) is a small (57cm in height) humanoid robot widely used to study robot-human relations and is specifically designed to be both expressive and approachable, especially for autistic children where, for example, it has been shown to stimulate social skills [59]. Conversely, Baxter (Figure 1), although having two arms and a simple animated face, is an industrial robot designed for the study of Human-Robot Collaboration (HRC) in tasks such as assembling and handling operations, as well as acting as a companion during the monitoring of worker safety.

The objects for the price judgment game were chosen after a screening test. We asked 20 participants to judge the price of 80 commonly-used objects, with 44 of these selected as stimuli for the experiment. Half of these stimulus (22 objects) were selected on the basis that participants would be uncertain how much they would cost, as demonstrated by relatively high variance between the price judgements ( $\mathrm{SD}=4.61)$. The remaining half of the stimulus (22 objects) had low price judgement variance $(\mathrm{SD}=0.69)$, meaning that the prices of these objects should be well known to participants. Stimuli from these two categories were equally split for application with either the Baxter or NAO robots so that for both robots participants were facing 22 objects, 11 low-price and 11 high-price variance. In order to exclude any differences in terms of price variance within the two robots, we performed statistical comparisons of the price variance for the objects used with

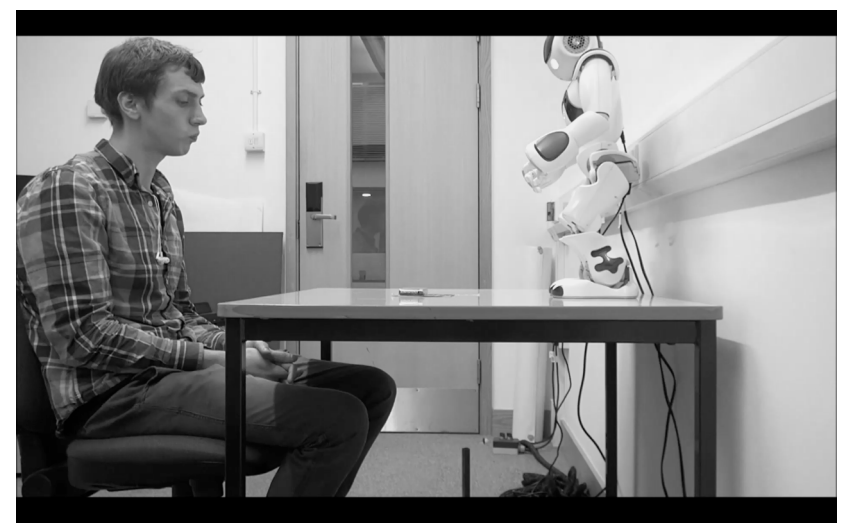

Figure 2 Experimental Setting.

the two robots, for both low and high variance condition. T-tests showed no significant differences for high $\left.t_{(20)}=0.599, p=.555\right)$ or low $\left(t_{(20)}=0.359, p=.722\right)$ price variance objects. All of the participants interacted with both Baxter and NAO robots, with the order of presentation counterbalanced between participants.

Moreover four questionnaires were used as secondary measures to the main experiment task. Three short scales measured Likeability, Trust and Credibility. The Likeability Questionnaire was based on Reysen [55]; the Trust scale was an adaptation of the Receptivity/Trust subscale of the Relational Communication Questionnaire and of the selection of Trust items in the IPIP International Personality Item Pool [29]. The Credibility scale was based on McCroskey and Young Source Credibility Scale [47]. In addition, we also used a questionnaire by Bartneck, Kulic, Croft and Zoghbi [8] typically used to measure a range of HRI factors (Anthropomorphism, Animacy, Likeability, Perceived Intelligence and Perceived Safety).

\subsubsection{Procedure}

Participants were seated at the same height as the robot, facing each other over a common table (Figure 2). In the preparatory phase of the experiment a series of objects were placed on the table between the robot and the participant by the experimenter. For each object the participants were asked to provide a description and two potential prices to the robot, which would then respond with a preference for one of the prices. After this preference was made the participants were asked to provide final feedback on whether they thought the price selected by the robot was correct. In these phase the description, prices, and correct price for the objects were provided to the participants on a written sheet. This part of the experiment was designed to provide a familiarisation with the main task, where the robot and human roles were reversed. 
In the main task an experimenter would place the stimulus object on the common table, and the robot would provide a brief verbal description of it, followed by two prices. Participants then had to make a choice between the two prices offered by the robot, after which the robot would give a positive or negative feedback on their selection. For the positive feedback the robot used to say "I agree with you", while nodding its head. For the negative one, the robot used to say "I do not agree", while shaking its head. If the robot had given negative feedback the participants were asked if they wanted to change their mind to agree with the choice of the robot, saying "Do you want to change your choice?". During this part of the experiment the robot could perform two different gaze behaviours (social and nonsocial). In the social gaze behaviour the robot looked first at the object on the table and, before starting describing the object, moved its gaze to the participants, while waiting for the participants to take a decision. In the nonsocial gaze behaviour the robot gaze was fixed at the stimulus for the whole time.

All participants were presented to the same predefined script of robot responses to the objects, which made no account of their own choice in the game. Participants completed 22 trials per robot and they were asked to complete the battery of questionnaires after the interaction with each robot.

The experiment was counterbalanced in a 2 (gaze behaviour: social or nonsocial) within subject design and a 2 (order: first or second) by 2 (robot: NAO or Baxter) betweesubject design.

\section{Results}

\subsection{Price Judgement Game results}

Social interactions with the robots were quantified through analysis of trials in which the robots disagreed with the price choice of the participants. A "changerate" was calculated as the proportion of these trials where the participants changed their original price selection to agree with the robot, compared to those in which they stuck with their original decision.

A forward stepwise linear mixed-effects model was fitted to the data, with change-rate as dependent variable, robot (NAO and Baxter), order (first or second robot presented) and gaze behaviour (social and nonsocial) as independent variables, and with participants as a random factor. Bonferroni's corrections were applied, when required. The overall effect size of the model was $r^{2}=0.56$. The model showed no significant main effects $\left(p_{s}>.05\right)$, but there was a significant interaction between robot and order $\left(X_{(3)}^{2}=10.93, p=.012\right)$.

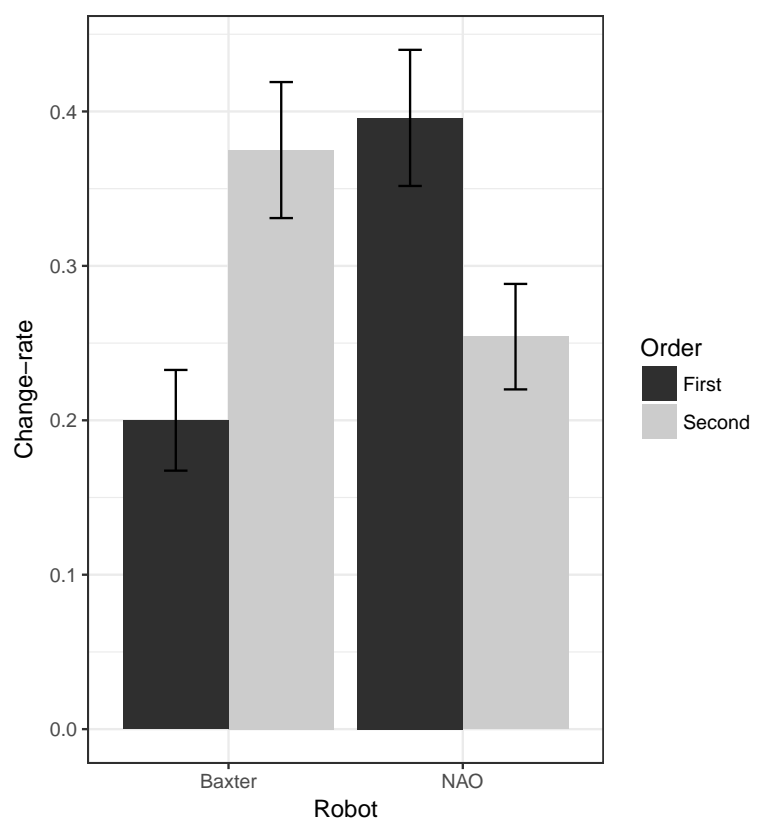

Figure 3 Two-way interaction between order and robot

Post hoc comparisons (Figure 3), revealed a higher change-rate for Baxter when participants interacted with it after the NAO robot, than when they interacted with it first $(p=.010)$. Conversely for the NAO robot, change-rates were lower when participants interacted with it after the Baxter robot $(p=.047)$. A comparison between robots in their first presentation also revealed that the NAO had a higher change-rate than Baxter $(p=.003)$. A significant interaction between robot and gaze behaviour $\left(X_{(2)}^{2}=14.77, p<.001\right)$ revealed a higher change-rate for the NAO robot when showing social gaze $(p=.036)$. For Baxter robot, gaze behaviour had no effect on change-rate $(p>.05)$. The three-way interaction between robot, order and gaze was also found to be significant $\left(X_{(2)}^{2}=11.83, p=.003\right)$. Post hoc comparisons (Figure 5) showed a significant effect of gaze only when the NAO was the first robot encountered by the participants $(p=.001)$. In this particular case social gaze increased the change-rate of participants. None of the other pairwise post hoc comparisons were significant $\left(p_{s}>.05\right)$.

\subsection{Questionnaires Results}

For the questionnaires, forward stepwise linear mixedeffects models were fitted to the data, with each scale as dependent measure, robot (NAO and Baxter) and order (first or second robot presented) as independent variables, and with participants as a random factor (Table 1). Bonferroni's corrections were applied, when 


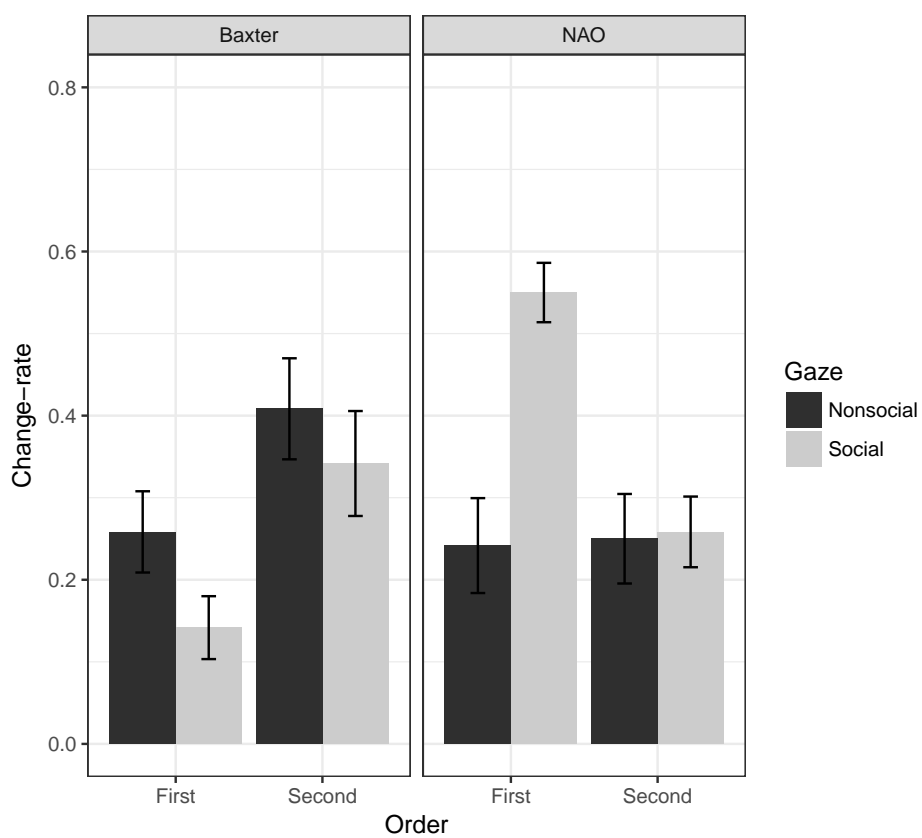

Figure 4 Three-way interaction between order, robot and gaze

Table 1 Questionnaires analysis results.

\begin{tabular}{lccc}
\hline & Order & Robot & Order by Robot \\
\hline \hline Likeability & n.s. & $X_{(1)}^{2}=25.24, p<.001$ & $X_{(2)}^{2}=12.15, p=.002$ \\
Trust & n.s. & $X_{(1)}^{2}=14.18, p<.001$ & n.s. \\
Credibility & n.s. & n.s. & n.s. \\
& & & \\
\hline Godspeed Questionnaires & & & \\
\hline Anthropomorphism & n.s. & $X_{(1)}^{2}=25.22, p<.001$ & $X_{(2)}^{2}=6.52, p=.038$ \\
Animacy & n.s. & $X_{(1)}^{2}=28.86, p<.001$ & $X_{(2)}^{2}=7.76, p=.021$ \\
Likeability & n.s. & $X_{(1)}^{2}=29.65, p<.001$ & $X_{(2)}^{2}=7.19, p=.027$ \\
Intelligence & n.s. & n.s. & n.s. \\
Safety & n.s. & $X_{(1)}^{2}=5.55, p=.018$ & n.s. \\
\hline
\end{tabular}

required. The type of robot had an effect for all the scales, with the exception for Credibility and Intelligence. Participants were overall preferring the NAO to the Baxter robot. Order had no effect on participants judgment. The two-way interaction between order and robot was significant for Likeability, Anthropomorphism and Animacy scales. Post hoc comparisons revealed that when presented after the more anthropomorphic NAO robot, Baxter had significantly higher ratings than when it was presented first (Figure 5) for all the four scales $\left(p_{s}<.050\right)$. For the NAO robot there were no significant effects of order for any of the four questionnaire scales $\left(p_{s}>.050\right)$.

\section{Discussion}

In this study we sought to understand one of the fundamental psychological properties of anthropomorphic
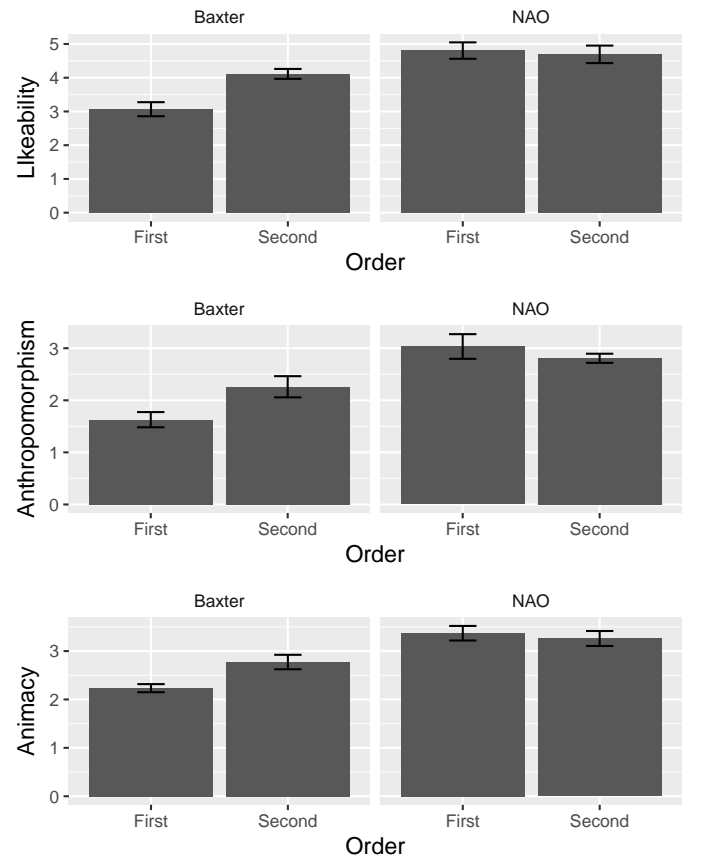

Figure 5 Two-way interaction between order and robot for Likeability, Anthropomorphism and Animacy scales.

projection, its specificity. When we anthropomorphise a non-human agent, we asked whether the assignment of that trait was strictly limited to that particular agent, or could also be generalised across other exemplars of the same type. Importantly, if there were variations in the distribution of the anthropomorphic features across a particular type of agent, would this category effect lead to us anthropomorphise exemplars that are missing the requisite features? We contended that the prior activation of a stereotype affects overt behaviour in a later encounter. To test this hypothesis we examined whether prior experience with a humanoid NAO robot would increase the credibility and anthropomorphism of a subsequently encountered Baxter robot, an industrial robot lacking many of the anthropomorphic features of the NAO. Importantly, if this was an effect of stereotype generalisation then we should also see the reverse effect if our first encounter was with Baxter robot. This initial encounter should de-anthropomorphise a subsequent encounter with the NAO robot.

We found that human-like form of the NAO robot anthropomorphised the Baxter robot, leading participants to associate a range of human-like traits and linked behaviours to it. Data from out questionnaires showed that participants rated the NAO robot as being more anthropomorphic than the Baxter robot, also rating it as being significantly more likeable, trusted, safe, and with greater animacy. However, we did not find any significant main effect between these robots in our 
primary measure, that of change-rate. This was the proportion of trials where participants changed their valuation of common objects to conform to the judgement of the robot, an implicit measure of the credibility of the robot. This provided an indirect measure of the degree of anthropomorphism associated with the robot, as it has been well established that human-like robots are perceived as more credible than less human-like variants $[21,32,66]$. Instead, we found the cross-over interaction required by our hypothesis. That is, the Baxter robot was perceived as being significantly more credible when participants interacted with it after the NAO robot. Conversely, the NAO robot was perceived as being more credible when participants interacted with it first, rather than after the Baxter. The questionnaire data also showed that experience with the NAO had a humanising effect on the Baxter, leading participants to rate the stereotypically-anthropomorphised Baxter higher for anthropomorphism, likeability, and animacy. However, questionnaire data for the NAO robot was not significantly affected by prior experience with Baxter. This disparity between an implicit measure of credibility seen in the change-rate of the NAO and the explicit post hoc rating provided by participants in the questionnaire is not unusual [35]. However, it does highlight the importance of implicit measures in the field of HRI where questionnaires, which may not always be sensitive to conceptual correspondence, are often the primary or sole source of behavioural data.

In addition to the study of anthropomorphic cues inherent to the form of the robots, we also examined the effect of human-like behaviour on the credibility of the robots. Both NAO and Baxter robots could engage in social or nonsocial gaze behaviour during the valuation task: moving their heads to engage in joint attention when discussing the valued object, or simply staring fixedly ahead. As this behaviour is orthogonal to the form of the robot we could also investigate interactions between anthropomorphic cues related to form and behaviour. Results showed that the effect of social gaze on credibility was limited to the NAO robot, and only when it had not been preceded by the Baxter. This interaction is in line with previous findings demonstrating that social gaze was human-like only prerogative [69] and did not provide an effective anthropomorphic cue; otherwise it should have had effect similar to that of form. Rather, that social gaze was only effective if participants had assigned human-like status to a robot. This is consistent with the activation of behavioural representations [20] or cognitive structures relating to interpersonal interactions [5] characteristic of the human-like trait. Other studies have shown that behaviour and interaction can be a more important cue to human-like status than form $[37,39]$. However, this result might be consistent with Guthrie's [31] psychological explanation of anthropomorphic projection. In contrast to the induction based explanation provided by Epley [23], Guthrie contends that anthropomorphism is not the result of our desire for simplified representation. Rather, it is the result of our seeking to project the most complex possible organisation onto any stimulus. As living intentional beings offer the greatest complexity, we seek to ascribe these properties onto any unknown agent as long as its features do not directly exclude them. If intentional social interactions, such as joint attention, were at a higher level of complexity than anthropomorphic form, any agent that violated the requirements of form would also exclude this higher level of attribution.

To conclude, we have shown that our anthropomorphic attribution to an agent, either as human or non-human, can affect subsequent encounters with similar agents. This stereotype activation means that our initial attributions tend to be generalised to other variants of similar type, even if they possess features that may be incongruent with that initial attribution. This extension of our understanding of the psychology of anthropomorphism has a growing application in the field in which we tested it, that of HRI. While robots are increasingly used in manufacturing industry, and have wide portrayal in the media [7], our own personal interactions with robots are extremely limited. This means that, for most of us, our first interaction with robots lies in the future, as does our potential anthropomorphism of these complex machines. Both the benefits and problems of anthropomorphism are particularly acute in robots, where familiarity [16] and believability [63] are keys to our acceptance of robots. Acceptance is a particular problem in health and adult social care, where robotic assistance has great potential in the care of the elderly [13]. Unfortunately, in this application, as in many others, the physical or technological constraints dictated by the required functions of a robot are often not compatible with a humanoid form. However, this study shows that if experience of more practical robots could be preceded with more anthropomorphic robots, then we would be more accepting of their appearance and behaviour.

\section{Compliance with Ethical Standards}

Funding: This study was funded by by the Air Force Office of Scientific Research, Air Force Materiel Command, USAF under Award No, FA9550-15-1-0025.

Disclosure of potential Conflict of Interest: The authors declare that they have no conflict of interest. 


\section{Research involving Human Participants and/or}

Animals: All procedures performed in studies involving human participants were in accordance with the ethical standards of the institutional and/or national research committee and with the 1964 Helsinki declaration and its later amendments or comparable ethical standards.

Informed consent: Informed consent was obtained from all individual participants included in the study.

\section{References}

1. Admoni, H., Scassellati, B.: Social eye gaze in humanrobot interaction: A review. J. Hum.-Robot Interact. 6(1), 25-63 (2017). DOI 10.5898/JHRI.6.1.Admoni. URL https://doi.org/10.5898/JHRI.6.1.Admoni

2. Aggarwal, P., McGill, A.L.: Is that car smiling at me? schema congruity as a basis for evaluating anthropomorphized products. Journal of Consumer Research 34(4), 468479 (2007)

3. Arnheim, R.: Visual thinking. Univ of California Press (1969)

4. Bae, J.e., Kim, M.s.: Selective visual attention occurred in change detection derived by animacy of robot's appearance. In: Collaboration Technologies and Systems (CTS), 2011 International Conference on, pp. 190-193. IEEE (2011)

5. Baldwin, M.W., Carrell, S.E., Lopez, D.F.: Priming relationship schemas: My advisor and the pope are watching me from the back of my mind. Journal of Experimental Social Psychology 26(5), 435-454 (1990)

6. Baron-Cohen, S.: Mindblindness: An essay on autism and theory of mind. MIT press (1997)

7. Bartneck, C.: Robots in the theatre and the media. Design \& semantics of form \& movement (DeSForM2013), Philips pp. 64-70 (2013)

8. Bartneck, C., Kulić, D., Croft, E., Zoghbi, S.: Measurement instruments for the anthropomorphism, animacy, likeability, perceived intelligence, and perceived safety of robots. International Journal of Social Robotics 1(1), 71-81 (2008). DOI 10.1007/s12369-008-0001-3

9. Baumeister, R.F., Leary, M.R.: The need to belong: desire for interpersonal attachments as a fundamental human motivation. Psychological bulletin 117(3), 497 (1995)

10. Bayliss, A.P., Tipper, S.P.: Predictive gaze cues and personality judgments: Should eye trust you? Psychological Science 17(6), 514-520 (2006)

11. Biernat, M., Manis, M., Nelson, T.E.: Stereotypes and standards of judgment. Journal of Personality and Social Psychology 60(4), 485 (1991)

12. Breazeal, C., Kidd, C.D., Thomaz, A.L., Hoffman, G., Berlin, M.: Effects of nonverbal communication on efficiency and robustness in human-robot teamwork. In: Intelligent Robots and Systems, 2005.(IROS 2005). 2005 IEEE/RSJ International Conference on, pp. 708-713. IEEE (2005)

13. Broadbent, E., Stafford, R., MacDonald, B.: Acceptance of healthcare robots for the older population: Review and future directions. International Journal of Social Robotics $\mathbf{1}(4), 319$ (2009)

14. Carey, S., Spelke, E.: Domain-specific knowledge and conceptual change. Mapping the mind: Domain specificity in cognition and culture 169, 200 (1994)

15. Cheney, D.L., Seyfarth, R.M.: How monkeys see the world: Inside the mind of another species. University of Chicago Press (1992)
16. Choi, J.g., Kim, M.: The usage and evaluation of anthropomorphic form in robot design. In: Undisciplined! Design Research Society Conference 2008 (2009)

17. Conard, N.J.: Palaeolithic ivory sculptures from southwestern germany and the origins of figurative art. Nature 426(6968), 830 (2003)

18. Dennet, D.C.: Kinds of minds: Towards an understanding of consciousness. Basic Books (1996)

19. Dennett, D.C.: The intentional stance. MIT press (1989)

20. Dijksterhuis, A., Van Knippenberg, A.: The relation between perception and behavior, or how to win a game of trivial pursuit. Journal of personality and social psychology 74(4), 865 (1998)

21. DiSalvo, C.F., Gemperle, F., Forlizzi, J., Kiesler, S.: All robots are not created equal: the design and perception of humanoid robot heads. In: Proceedings of the 4th conference on Designing interactive systems: processes, practices, methods, and techniques, pp. 321-326. ACM (2002)

22. Dittrich, W.H., Lea, S.E.: Visual perception of intentional motion. Perception 23(3), 253-268 (1994)

23. Epley, N., Waytz, A., Cacioppo, J.T.: On seeing human: a three-factor theory of anthropomorphism. Psychological review 114(4), 864 (2007)

24. Fong, T., Nourbakhsh, I., Dautenhahn, K.: A survey of socially interactive robots. Robotics and autonomous systems 42(3-4), 143-166 (2003)

25. Freire, A.L., Barreto, G.A., Veloso, M., Varela, A.T.: Shortterm memory mechanisms in neural network learning of robot navigation tasks: A case study. In: Robotics Symposium (LARS), 2009 6th Latin American, pp. 1-6. IEEE (2009)

26. Fussell, S.R., Kiesler, S., Setlock, L.D., Yew, V.: How people anthropomorphize robots. In: Proceedings of the 3rd ACM/IEEE international conference on Human robot interaction, pp. 145-152. ACM (2008)

27. Garau, M., Slater, M., Bee, S., Sasse, M.A.: The impact of eye gaze on communication using humanoid avatars. In: Proceedings of the SIGCHI conference on Human factors in computing systems, pp. 309-316. ACM (2001)

28. Goetz, J., Kiesler, S., Powers, A.: Matching robot appearance and behavior to tasks to improve human-robot gration. In: Robot and Human Interactive Communication, 2003. Proceedings. ROMAN 2003. The 12th IEEE International Workshop on, pp. 55-60. Ieee (2003)

29. Goldberg, L.R., Johnson, J.A., Eber, H.W., Hogan, R., Ashton, M.C., Cloninger, C.R., Gough, H.G.: The international personality item pool and the future of publicdomain personality measures. Journal of Research in personality 40(1), 84-96 (2006)

30. Graham, S.A., Poulin-Dubois, D.: Infants' reliance on shape to generalize novel labels to animate and inanimate objects. Journal of Child Language 26(2), 295-320 (1999)

31. Guthrie, S.E., Guthrie, S.: Faces in the clouds: A new theory of religion. Oxford University Press on Demand (1993)

32. Hancock, P.A., Billings, D.R., Schaefer, K.E., Chen, J.Y.C., De Visser, E.J., Parasuraman, R.: A meta-analysis of factors affecting trust in human-robot interaction. Human Factors: The Journal of the Human Factors and Ergonomics Society 53(5), 517-527 (2011)

33. Hauser, M.: Wild minds: What animals really think. Macmillan (2001)

34. Heider, F., Simmel, M.: An experimental study of apparent behavior. The American journal of psychology 57(2), 243259 (1944)

35. Hofmann, W., Gawronski, B., Gschwendner, T., Le, H., Schmitt, M.: A meta-analysis on the correlation between 
the implicit association test and explicit self-report measures. Personality and Social Psychology Bulletin 31(10), 1369-1385 (2005)

36. Huang, C.M., Thomaz, A.L.: Joint attention in humanrobot interaction. In: AAAI Fall Symposium: Dialog with Robots (2010)

37. Kahn Jr, P.H., Ishiguro, H., Friedman, B., Kanda, T., Freier, N.G., Severson, R.L., Miller, J.: What is a human?: Toward psychological benchmarks in the field of humanrobot interaction. Interaction Studies 8(3), 363-390 (2007)

38. Kennedy, J.S.: The new anthropomorphism. Cambridge University Press (1992)

39. Kiesler, S., Goetz, J.: Mental models of robotic assistants. In: CHI'02 extended abstracts on Human Factors in Computing Systems, pp. 576-577. ACM (2002)

40. Kiesler, S., Powers, A., Fussell, S.R., Torrey, C.: Anthropomorphic interactions with a robot and robot-like agent. Social Cognition 26(2), 169-181 (2008)

41. Kim, S., McGill, A.L.: Gaming with mr. slot or gaming the slot machine? power, anthropomorphism, and risk perception. Journal of Consumer Research 38(1), 94-107 (2011)

42. Krach, S., Hegel, F., Wrede, B., Sagerer, G., Binkofski, F., Kircher, T.: Can machines think? interaction and perspective taking with robots investigated via fmri. PloS one $\mathbf{3}(7)$, e2597 (2008)

43. Kuchenbrandt, D., Eyssel, F., Bobinger, S., Neufeld, M.: Minimal group-maximal effect? evaluation and anthropomorphization of the humanoid robot nao. In: International Conference on Social Robotics, pp. 104-113. Springer (2011)

44. Lesher, J.H.: Xenophanes of Kolophon. Wiley Online Library $(1992)$

45. Leyens, J.P., Yzerbyt, V., Schadron, G.: Stereotypes and social cognition. Sage Publications, Inc (1994)

46. Mason, M.F., Tatkow, E.P., Macrae, C.N.: The look of love: Gaze shifts and person perception. Psychological Science 16(3), 236-239 (2005)

47. McCroskey, J.C., Young, T.J.: Ethos and credibility: The construct and its measurement after three decades. Communication Studies 32(1), 24-34 (1981)

48. Mitchell, R.W., Hamm, M.: The interpretation of animal psychology: anthropomorphism or behavior reading? Behaviour 134(3), 173-204 (1997)

49. Morewedge, C.K., Preston, J., Wegner, D.M.: Timescale bias in the attribution of mind. Journal of personality and social psychology 93(1), 1 (2007)

50. Mutlu, B., Shiwa, T., Kanda, T., Ishiguro, H., Hagita, N.: Footing in human-robot conversations: how robots might shape participant roles using gaze cues. In: Proceedings of the 4th ACM/IEEE international conference on Human robot interaction, pp. 61-68. ACM (2009)

51. Nass, C., Brave, S.: Wired for speech: How voice activates and advances the human-computer relationship. MIT press (2005)

52. Nass, C., Isbister, K., Lee, E.J., et al.: Truth is beauty: Researching embodied conversational agents. Embodied conversational agents pp. 374-402 (2000)

53. Powers, A., Kramer, A.D., Lim, S., Kuo, J., Lee, S.l., Kiesler, S.: Eliciting information from people with a gendered humanoid robot. In: Robot and Human Interactive Communication, 2005. ROMAN 2005. IEEE International Workshop on, pp. 158-163. IEEE (2005)

54. Rau, P.P., Li, Y., Li, D.: Effects of communication style and culture on ability to accept recommendations from robots. Computers in Human Behavior 25(2), 587-595 (2009)

55. Reysen, S.: Construction of a new scale: The Reysen likability scale. Social Behavior and Personality: an international journal 33(2), 201-208 (2005)
56. Riek, L.D., Rabinowitch, T.C., Chakrabarti, B., Robinson, P.: How anthropomorphism affects empathy toward robots. In: Proceedings of the 4th ACM/IEEE international conference on Human robot interaction, pp. 245-246. ACM (2009)

57. Riva, P., Sacchi, S., Brambilla, M.: Humanizing machines: Anthropomorphization of slot machines increases gambling. Journal of Experimental Psychology: Applied 21(4), 313 (2015)

58. Salem, M., Rohlfing, K., Kopp, S., Joublin, F.: A friendly gesture: Investigating the effect of multimodal robot behavior in human-robot interaction. In: RO-MAN, 2011 IEEE, pp. 247-252. IEEE (2011)

59. Shamsuddin, S., Yussof, H., Ismail, L.I., Mohamed, S., Hanapiah, F.A., Zahari, N.I.: Initial response in hri-a case study on evaluation of child with autism spectrum disorders interacting with a humanoid robot nao. Procedia Engineering 41, 1448-1455 (2012)

60. Staudte, M., Crocker, M.W.: Investigating joint attention mechanisms through spoken human-robot interaction. Cognition 120(2), 268-291 (2011)

61. Syrdal, D.S., Dautenhahn, K., Koay, K.L., Walters, M.L., Ho, W.C.: Sharing spaces, sharing lives-the impact of robot mobility on user perception of a home companion robot. In: International Conference on Social Robotics, pp. 321-330. Springer (2013)

62. Tam, K.P., Lee, S.L., Chao, M.M.: Saving mr. nature: Anthropomorphism enhances connectedness to and protectiveness toward nature. Journal of Experimental Social Psychology 49(3), 514-521 (2013)

63. Tapus, A., Mataric, M.J., Scassellati, B.: Socially assistive robotics [grand challenges of robotics]. IEEE Robotics \& Automation Magazine 14(1), 35-42 (2007)

64. Tremoulet, P.D., Feldman, J.: Perception of animacy from the motion of a single object. Perception 29(8), 943-951 (2000)

65. Walters, M.L., Koay, K.L., Syrdal, D.S., Dautenhahn, K., Te Boekhorst, R.: Preferences and perceptions of robot appearance and embodiment in human-robot interaction trials. Procs of New Frontiers in Human-Robot Interaction (2009)

66. Waytz, A., Heafner, J., Epley, N.: The mind in the machine: Anthropomorphism increases trust in an autonomous vehicle. Journal of Experimental Social Psychology 52, 113-117 (2014)

67. Wichman, A.L.: Uncertainty threat can cause stereotyping: The moderating role of personal need for structure. Sage Open 2(2), 2158244012444442 (2012)

68. Yee, N., Bailenson, J.N., Rickertsen, K.: A meta-analysis of the impact of the inclusion and realism of human-like faces on user experiences in interfaces. In: Proceedings of the SIGCHI conference on Human factors in computing systems, pp. 1-10. ACM (2007)

69. Zanatto, D., Patacchiola, M., Goslin, J., Cangelosi, A.: Priming anthropomorphism: Can the credibility of humanlike robots be transferred to non-humanlike robots? In: Human-Robot Interaction (HRI), 2016 11th ACM/IEEE International Conference on, pp. 543-544. IEEE (2016)

70. Złotowski, J., Proudfoot, D., Yogeeswaran, K., Bartneck, C.: Anthropomorphism: opportunities and challenges in human-robot interaction. International Journal of Social Robotics 7(3), 347-360 (2015) 